\title{
Comunicación de riesgo en salud por la COVID-19 desde la percepción de los pobladores de Arequipa en 2021
}

José Arias Gonzáles

Universidad Católica de Santa María joseariasgon6@gmail.com

ORCID: https://orcid.org/0000-0002-3250-5287

Tania Luz Tafur Pittman

Universidad Nacional José Faustino Sánchez Carrión

taniatafur@gmail.com

ORCID: https://orcid.org/0000-0002-4370-090X

\section{Gloria María Delgado Suaña}

Universidad Andina del Cusco

gloriamaria.delgados@gmail.com

ORCID: https://orcid.org/0000-0002-0690-1323

Fecha de finalización: 18 de agosto de 2021. Fecha de envío: 19 de agosto de 2021.

Fecha de aceptación: 17 de noviembre de 2021. DOI: https://doi.org/10.26422/aucom.2021.1002.nav

\section{Resumen}

El protagonismo de los medios de comunicación en situaciones de riesgo para la salud, como es el caso de la COVID-19, se evidencia con el incremento del interés de la población por mantenerse informada y por las noticias actualizadas relacionadas con estos acontecimientos. El estudio tuvo como objetivo general evaluar la comunicación de riesgo en salud por la COVID-19 desde la percepción de los pobladores de la región de Arequipa, Perú, en 2021. Se realizó un estudio básico con un alcance descriptivo y un diseño no experimental. La población se conformó por 1316000 habitantes de esta región; luego de usar el muestreo probabilístico-aleatorio simple, la muestra quedó constituida por 384 habitantes. La técnica utilizada fue la encuesta y el instrumento fue el cuestionario. La mayoría de los mensajes emitidos por las organizaciones gubernamentales de Perú han generado niveles bajos de confianza en los pobladores, percibiendo una falta de credibilidad, actualidad, información oportuna y transparencia, además de mensajes hilarantes y con doble sentido que no orientan a las personas a evitar el contagio. La importancia de escuchar a la población y de planificar las acciones no ha sido tomada en cuenta por parte de las organizaciones responsables y los medios de comunicación, lo cual ha repercutido en la desatención de los pobladores, provocando una negativa comunicación de riesgo en salud por la COVID-19.

Palabras clave: comunicación de riesgo, COVID-19, medios de comunicación, redes sociales, salud pública. 


\title{
The communication of COVID-19 health risks, from the perspective of the population of Arequipa in 2021
}

\begin{abstract}
In health risk situations, communication media play an increasingly important role. This became evident during the COVID-19 pandemic, as populations looked to the media to remain informed and updated. This study aims to evaluate the communication of COVID-19 health risks, from the point of view of the population of the Arequipa region in Peru during 2021. We carried out a basic study with a descriptive scope and a non-experimental design. The region has a population of $1,316,000$. Following a simple random sampling, we surveyed 384 inhabitants. We found that most communications sent out by Peru's public institutions had failed to inspire confidence in the local population. They perceived a lack of credibility, timeliness, and transparency. To make matters worse, some of these messages even came across as humorous, with double entendres that did not help people avoid contagion. Communication media and public institutions evidently did not consider the importance of listening to the population and appropriately planning their actions. This has led to populations not being properly cared for and an overall negative perception of health risk communications during the pandemic.
\end{abstract}

Keywords: health risk communication, COVID-19, communications media, social media, public health.

\section{Comunicação de risco à saúde devido à COVID-19 na percepção dos habitantes de Arequipa, 2021}

\section{Resumo}

O destaque da mídia em situações de risco para a saúde como a COVID-19 é evidenciado pelo aumento do interesse da população em se manter informada e por notícias atualizadas relacionadas a esses eventos. O objetivo geral do estudo foi avaliar a comunicação de risco à saúde pela COVID-19 a partir da percepção dos moradores da região de Arequipa, 2021. Foi realizado um estudo básico com âmbito descritivo e delineamento não experimental. A população foi constituída por 1 milhão 316 de habitantes da cidade de Arequipa, por meio de amostragem probabilística-aleatória simples, a amostra foi composta por 384 habitantes da cidade de Arequipa. A técnica utilizada foi a survey e o instrumento foi o questionário. A maior parte das mensagens emitidas por órgãos governamentais do Peru têm gerado baixos níveis de confiança nos habitantes, percebendo falta de credibilidade, oportunidade, informação oportuna e transparência, bem como mensagens com duplo sentido e hilariantes que não orientam as pessoas a evitar o contágio. A importância de ouvir a população e planejar ações não tem sido levada em consideração pelos órgãos responsáveis e pela mídia, o que tem impactado a desatenção dos moradores, causando uma comunicação negativa de risco à saúde por parte da COVID-19.

Palavras chave: comunicação de risco, COVID-19, meios de comunicação, redes sociais, saúde pública.

\section{Introducción}

A nivel mundial, los Gobiernos han desarrollado políticas informativas y los servicios de salud y organizaciones no gubernamentales han generado información y campañas publicitarias para que la comunidad conozca las medidas preventivas contra la COVID-19 (Brajkovic et al., 2020). Pese a la información que circula sobre esta enferme- 


\section{Austral@municación \\ Volumen 10, número 2 (Diciembre de 2021): 427-440 ISSN (I) 2313-9129. ISSN (E) 2313-9137}

dad, una parte de la población no respeta las normas sanitarias, como el distanciamiento social o el uso de mascarillas, generando un aumento de infectados y fallecidos, así como la saturación en los servicios de salud (Peña-López y Rincón-Orozc, 2020). La otra parte guarda las medidas de seguridad, pero se infecta por terceros que son portadores sanos o que se encuentran en el periodo "ventana" de la infección (Lázaro-Rodríguez y HerreraViedma, 2020). Entre las razones de este comportamiento de negación se encuentra la falta de creencias en la información que brindan los medios de comunicación (Mejia et al., 2020). Para febrero de 2020, la cantidad de artículos publicados con información relacionada con la pandemia fue 23 veces superior a los artículos sobre el ébola en agosto 2018. De acuerdo con algunas investigaciones en España, para abril de 2020 se han publicado más de 432058 noticias referentes a la pandemia. En un entorno de infodemia, el rol que cumplen los medios de comunicación en situaciones de crisis sanitarias es el más relevante, pues estos se encargan de emitir los mensajes oficiales e información a los ciudadanos sobre las medidas preventivas (Masip et al., 2020).

Justamente, dentro de las estrategias para hacer frente a este virus y su propagación se encuentran los anuncios informativos (Rosas y Barrios, 2017) a partir del uso de los medios de comunicación, como prensa escrita, radio y televisión y, en particular, el uso del teléfono móvil y las redes sociales para informar a la población y promover comportamientos sanitarios a través de campañas (Feenstra y Pallarés-Domínguez, 2017). En esta pandemia, los mensajes no han sido diseñados de acuerdo al perfil de la audiencia ni por los canales adecuados en un contexto de emergencia sanitaria (LázaroRodríguez y Herrera-Viedma. 2020). Además, circulan noticias falsas en las redes sociales que generan confusión e incertidumbre (Martínez, 2017). Como resultado, los segmentos de la población no se informan adecuadamente y asumen comportamientos que ponen en riesgo su vida (García-Áviles et al. 2014).

En el Perú, debido a la información que brindan los medios de comunicación, las conductas de la población se tornaron excesivas; por ejemplo, se dijo que beber mucha agua ayudaría a prevenir o desaparecer el virus, y un tuit publicado por el Ministro de Salud de Francia que llegó al Perú a través de las redes sociales anunció que el ibuprofeno tenía una relación positiva sobre el virus. Se empezó a comprar de forma irracional papel higiénico y productos no perecederos, dejando desabastecidos los establecimientos y, de forma indirecta, a los otros pobladores (Gobierno Regional de Arequipa, 2021). Estas noticias, que se difundían y aún se siguen difundiendo, carecen de sustento científico y generan incertidumbre, temor, panico y hasta algunos efectos negativos en la salud. Dado que la comunicación es una extensión constitutiva en los procesos de la salud, se presenta en situaciones de emergencia y guarda relación con el riesgo que tiene la comunicación y la percepción de las personas sobre los peligros a los que pueden estar expuetos (Gómez, 2017). 
José Arias Gonzáles, Tania Luz Tafur Pittman, Gloria María Delgado Suaña

Comunicación de riesgo en salud por la COVID-19 desde la percepción de los pobladores de Arequipa en 2021

Estudios realizados desde este enfoque, como el de Gonzáles et al. (2021) sobre la percepción de riesgo por la COVID-19 en pobladores de Manzanilllo (México), concluyó en una inadecuada percepción de riesgo que se debió a los bajos niveles de susceptibilidad, severidad y autoeficacia para llevar a cabo acciones de comunicación para evitar la enfermedad por parte de instituciones del Gobierno y medios de comunicación. En la investigación realizada por Hernández et al. (2021), se evidenció la necesidad de una comunicación efectiva que brinde información veraz y clara, acompañada de solidaridad, optimismo y fe en la recuperación de la salud, así como de una sensibilidad intercultural que favorezca la comprensión de determinadas actitudes y prácticas no comunes y permita un mejor desempeño profesional. Por otro lado, Orozco (2020) presentó un trabajo de investigación sobre las estrategias de comunicación del Gobierno del Perú durante el estado de emergencia por la COVID-19, evidenciando que la población tuvo una aprobación de los mensajes emitidos durante la primera fase de la campaña, no obstante, en las semanas posteriores, esa percepción cambió de manera significativa, pues se lograron vislumbrar algunos indicios de rechazo por parte de la población, lo que permitió afirmar que las estrategias de comunicación utilizadas no fueron lo suficientemente efectivas y que el mensaje no fue entendido por la población. Por su parte, García (2017) presentó un estudio sobre la comunicación de crisis en los gabinetes de prensa sanitarios, en el cual muestra que, en diversas ocasiones, se compartieron datos de la vida privada y médica de los afectados. Asimismo, se contó con exceso de información por parte de las más de 605 noticias publicadas durante la crisis sanitaria. En tal sentido, la información divulgada durante ese período, a pesar de ser rápida, muchas veces carecía de calidad y era algo excesiva.

Como se expuso líneas arriba, la crisis sanitaria originada por la COVID-19 ha provocado no solo el colapso de los sistemas de salud, sino también una extensa perturbación social en la población, por lo que conocer la percepción del riesgo en salud permitirá establecer una adecuada comunicación de riesgo a través de la emisión de mensajes de salud pública efectivos, la identificación de los canales adecuados y redes de influencia que faciliten una respuesta positiva y de calidad a la población en situaciones de riesgo. Por tal razón, el presente artículo científico tiene como objetivo general determinar la percepción de la comunicación de riesgo en salud por la COVID-19 de los pobladores de la región de Arequipa en 2021.

\section{Rol de los medios de comunicación}

El rol que cumplen los medios de comunicación en situaciones de emergencias de salud ha sido estudiado en diversos trabajos científicos. Los periodistas y el personal de salud comparten una analogía durante el brote de alguna enfermedad: ambos grupos cumplen una función importante en relación con la información, con la percepción de 


\section{Austral Omunicación \\ Volumen 10, número 2 (Diciembre de 2021): 427-440 ISSN (I) 2313-9129. ISSN (E) 2313-9137}

la población y la toma de decisiones de los entes responsables del control (Masip et al., 2020). El propósito de los periodistas es informar con responsabilidad para contribuir a la prevención de la enfermedad, disminuyendo los índices de ansiedad, incertidumbre y pánico. Es un desafío para el periodismo anticiparse a los acontecimientos con la rapidez de la difusión de información incorrecta (Lázaro-Rodríguez y Herrera-Viedma, 2020).

El protagonismo de los medios en situaciones de riesgos de salud, como es el caso de las pandemias, se evidencia cuando en situaciones de alerta provocadas por situaciones de salud o catástrofes se incrementa el interés de la población por mantenerse informada y por las noticias actualizadas relacionadas con esos acontecimientos. Todo esto produce una alteración en el uso de los medios de comunicación. De allí la importancia de los medios de comunicación en las situaciones de alerta o de riesgo de salud (Lázaro-Rodríguez y Herrera-Viedma, 2020).

La pandemia que azota al mundo es una emergencia sanitaria y, en la actualidad, existe un gran número de contagiados y una cantidad considerable de fallecidos, además del impacto económico y social que ha generado. Por ello, los medios de comunicación tienen el rol de informar de manera correcta a la población y ofrecer los datos que contribuyan a la toma de buenas decisiones, por medio de la veracidad y la calidad de la información brindada. El buen uso del medio contribuirá a la comprensión y aplicación de medidas preventivas. Es importante tener en cuenta que entre la población se puede generar información exagerada, falsa o distorsionada, por lo que es indispensable siempre emitir información con sustento científico (Mejía et al., 2020).

\section{Comunicación de riesgo}

La comunicación de riesgo constituye un conjunto de actividades esenciales para salvar vidas desde diversos contextos. Esta actividad se ha desarrollado para evitar o controlar situaciones de riesgo, como accidentes de tránsito, alimentación saludable, problemas con el medio ambiente, desastres naturales y diversas enfermedades (Ministerio de Salud de Chile, 2020). La comunicación de riesgo se considera un proceso interactivo de intercambio de información u opiniones entre las personas y las instituciones, en el cual se da un mensaje relacionado con el riesgo (Rodríguez, 2019).

La comunicación de riesgo puede clasificarse de acuerdo con la temática, pero teniendo en cuenta que es una manera de persuadir a las personas que se encuentran ante un riesgo inminente (Organización Panamericana de la Salud, 2011), la comunicación se emplea como un elemento integrador por medio del cual se implementan y desarrollan un conjunto de planes y programas que ayuden al colectivo social a afrontar los riesgos percibidos (Rosas y Barrios, 2017). 
José Arias Gonzáles, Tania Luz Tafur Pittman, Gloria María Delgado Suaña

Comunicación de riesgo en salud por la COVID-19 desde la percepción de los pobladores de Arequipa en 2021

\title{
Comunicación de riesgo en salud
}

La comunicación de riesgo en salud se desarrolló a partir de las experiencias en el ámbito sanitario presididas por la Organización Mundial de la Salud (OMS), en la que sobresalen las estrategias de comunicación de riesgo como un mecanismo fundamental para disminuir las consecuencias de las emergencias sanitarias. Por ello, la comunicación de riesgo es el desarrollo teórico-práctico que ha adquirido la influencia de diversas teorías, modelos y puntos de vista de la sociología, la psicología, la economía y la educación social, solo por hacer mención a algunas referencias en el ámbito científico que han impactado en la práctica sanitaria en función de la prevención de los riesgos de la salud (Gómez, 2017).

Algunos países dan un dispositivo de salud pública de monitoreo, vigilancia epidemiológica, administración y comunicación de riesgo para dar respuesta a los retos que demanda la salud pública. El asunto de comunicación de riesgo en salud es de suma importancia, especialmente cuando se trata de epidemias y pandemias, como la que actualmente atraviesa la humanidad (Prior, 2020). Entre los principales medios utilizados para la comunicación de riesgo en el ámbito de la salud se encuentran: divulgación de comunicados diarios, organización de conferencias de prensa, producción de información para sitios web y redes sociales, producción de material de comunicación, redes sociales y uso de canales telefónicos de atención (Rodríguez, et al. 2018).

De acuerdo con Gómez (2017), la comunicación de riesgo es

\begin{abstract}
El proceso de toma de decisiones que toma en cuenta los aspectos políticos, sociales y económicos que ven el riesgo como un elevado o inminente peligro, por ende se encargan de plantar, estudiar y comparar alternativas de control para escoger la mejor respuesta que le pueda brindar seguridad a la población ante un posible peligro, este le da una enorme relevancia al diálogo con el colectivo social afectado y las demás personas interesadas, para aportar información relevante, que les permita determinar las mejores decisiones durante una emergencia o desastre relacionado con la salud. (p. 5)
\end{abstract}

Según la OMS, existen seis factores de comunicación de riesgo que los Gobiernos deben tener en cuenta para hacer frente a situaciones de vulnerabilidad en los pobladores; la comunicación debe ser: fácil de entender, accesible, factible, creíble, pertinente y a tiempo. Estos factores permiten que la población pueda protegerse frente a situaciones de riesgo. De la misma manera, la comunicación de riesgo en salud se encuentra unida a la confianza, los anuncios tempranos, la transparencia, escuchar al público y la planificación (Gómez, 2017)

\section{Metodología}

El enfoque de investigación fue cuantitativo. Según Hernández-Sampieri y Mendoza (2018), este estudio se realiza bajo características numéricas -los resultados 


\section{Austral Omunicación \\ Volumen 10, número 2 (Diciembre de 2021): 427-440 ISSN (I) 2313-9129. ISSN (E) 2313-9137}

van a permitir cuantificar la información- y se usan herramientas estadísticas para los resultados. El diseño de investigación fue no experimental, ya que no se van a manipular las variables y serán estudiadas en un contexto natural. Además, es transversal, debido a que se recopila la información en un único periodo (Arias, 2020a). El nivel de investigación fue descriptivo, ya que el investigador describirá el fenómeno que está investigando; asimismo, se busca caracterizar propiedades, rasgos, situaciones o eventos de la población participante del estudio (Hernández-Sampieri y Mendoza, 2018). El tipo de investigación fue básico debido a que el objetivo principal es ahondar en los huecos del conocimiento a fin de reconocer la problemática de forma teórica y científica (Hernández-Sampieri y Mendoza, 2018). El estudio no presentó hipótesis porque pretende describir y reconocer de forma inicial la percepción de los pobladores del distrito en cuanto a la comunicación de riesgo en salud por la COVID-19.

La unidad de análisis se conformó por 32 anuncios sobre medidas para prevenir la COVID-19 publicadas en Facebook por las páginas oficiales de la Presidencia de la República del Perú, la Policía Nacional del Perú y el Ministerio de Salud del Perú en un periodo comprendido entre febrero y abril de 2021. La población y unidad de muestreo estuvieron conformadas por 1316000 pobladores en la región de Arequipa. Para hallar la muestra, se utilizó el método probabilístico-aleatorio simple, para lo cual se aplicó un muestreo estadístico con un nivel de confianza del 95\% y un margen de error del 5\%. La muestra quedó conformada por 384 habitantes de esta región. Para constituir la unidad de análisis, se utilizaron los siguientes criterios por conveniencia: participantes de entre 18 y 60 años y participantes que no trabajen en organizaciones gubernamentales encargadas de difundir anuncios para prevenir la COVID-19 o sean parte de los medios de comunicación. Para acceder a la muestra, fuimos a centros comerciales y entidades financieras de la ciudad de Arequipa, donde se abordaron a todos los participantes de la muestra. Con respecto a los datos sociodemográficos, la muestra se segmentó en 218 hombres y 166 mujeres, con edades de entre 18 y 25 años (8\%), 26 y 30 años (19\%), 31 y 40 años (61\%), 41 y 50 años (9\%) y 51 y 60 años (3\%).

Para medir la variable se utilizó la técnica de la encuesta, que, según Arias (2020b), es una herramienta que permite evaluar las características, comportamientos o percepciones de un grupo de personas. Como instrumento se utilizó el cuestionario, que consiste en un conjunto de preguntas presentadas y enumeradas en una tabla y una serie de posibles respuestas que el encuestado debe responder. No existen respuestas correctas o incorrectas, todas llevan a un resultado diferente y se aplican a una población conformada por personas (Arias, 2021).

El instrumento fue elaborado por los autores del estudio y estuvo conformado por 18 preguntas que midieron 5 dimensiones de la comunicación de riesgo en salud, como confianza de los anuncios (credibilidad y distorsión de los anuncios), anuncios 
José Arias Gonzáles, Tania Luz Tafur Pittman, Gloria María Delgado Suaña

Comunicación de riesgo en salud por la COVID-19 desde la percepción de los pobladores de Arequipa en 2021

tempranos (información actualizada, anuncios oportunos), trasparencia de los anuncios (información alarmante, claridad de los mensajes), escuchar al público (opinión del público, medidas contra la COVID-19) y planificación de los anuncios (mensajes con objetivos claros, medidas planificadas contra la COVID-19). Se tuvieron en cuenta un total de 32 anuncios de las tres organizaciones gubernamentales y se utilizó la escala tipo Likert con una codificación de: en desacuerdo (1), indiferente (2) y de acuerdo (3), además de una calificación para las cinco dimensiones de: nivel alto, nivel regular, nivel bajo. Se validó por medio de juicio de expertos bajo el método V de Aiken y se tuvo como conclusión que el instrumento fue válido con un valor de .89 y mediante una prueba piloto para someterlo al método de Alpha de Cronbach, concluyendo que el instrumento fue válido y confiable con un valor obtenido de .806. El cuestionario se aplicó la segunda semana del mes de mayo con una duración de seis días.

Para obtener los resultados del estudio se aplicaron los instrumentos en físico y posteriormente se contabilizaron y codificaron para sistematizarlas en Excel. Luego, se generaron las tablas de distribución con frecuencia absoluta (frecuencia) y frecuencia relativa (porcentaje) para luego exportarlo a Word y realizar su interpretación y análisis. Los resultados obtenidos para la investigación no serán divulgados de forma que afecte a los participantes, a quienes tampoco se les pidió información personal. Para ello, se les presentó un consentimiento informado a los participantes a fin de poner de manifiesto esta información. Asimismo, todo el contenido de esta investigación se sustenta en bases empíricas y originales.

\section{Resultados}

Como se muestra en la Tabla 1, el $88 \%$ de los pobladores encuestados tiene un nivel bajo de confianza en los anuncios publicados por las organizaciones gubernamentales del Perú. Al no registrarse este vínculo entre pobladores y las organizaciones, es poco probable que las personas sigan las recomendaciones para evitar el contagio, ya que perciben que los mensajes carecen de credibilidad.

Tabla 1. Nivel de confianza de los anuncios publicados por las organizaciones gubernamentales según la percepción de los pobladores

\begin{tabular}{lcc}
\hline & Frecuencia & Porcentaje \\
\hline Alto & 19 & $5 \%$ \\
Regular & 26 & $7 \%$ \\
Bajo & 339 & $88 \%$ \\
\hline
\end{tabular}

De acuerdo con la Tabla 2, el 51\% de los pobladores encuestados percibe los anuncios publicados por las organizaciones gubernamentales del Perú con un nivel bajo de 
Austral Comunicación

Volumen 10, número 2 (Diciembre de 2021): 427-440

ISSN (I) 2313-9129. ISSN (E) 2313-9137

actualidad. Más de la mitad de los pobladores consigna que la información recibida no es oportuna, ya que, más que alertar para que se mantengan preparados, crean pánico $\mathrm{y}$ una eventual desinformación sobre las medidas para prevenir el contagio.

Tabla 2. Nivel de actualidad de los anuncios publicados por las organizaciones gubernamentales según la percepción de los pobladores

\begin{tabular}{lcc}
\hline & Frecuencia & Porcentaje \\
\hline Alto & 83 & $21 \%$ \\
Regular & 106 & $28 \%$ \\
Bajo & 195 & $51 \%$ \\
\hline
\end{tabular}

Según la Tabla 3, el 74\% de los pobladores encuestados percibe que los anuncios publicados por las organizaciones gubernamentales del Perú tienen un nivel bajo de transparencia. La mayoría precisa que la información que le brindan es distorsionada, haciéndola creer en realidades distintas a las vividas. En vez de persuadir para que los pobladores eviten el contagio, pone en juego el derecho de las personas de recibir información veraz y de calidad.

Tabla 3. Nivel de transparencia de los anuncios publicados por las organizaciones gubernamentales según la percepción de los pobladores

\begin{tabular}{lcc}
\hline & Frecuencia & Porcentaje \\
\hline Alto & 39 & $10 \%$ \\
Regular & 62 & $16 \%$ \\
Bajo & 283 & $74 \%$ \\
\hline
\end{tabular}

Conforme con la Tabla 4, el 86\% de los pobladores encuestados precisan que no han sido escuchados por parte de las organizaciones gubernamentales del Perú. Las costumbres, creencias, comportamientos y hábitos de las personas de esta ciudad no fueron tomados en cuenta para establecer disposiciones con el fin de evitar el contagio. Los niveles bajos de empatía e identificación con los pobladores han sido fundamentales para que la comunicación de riesgo en salud sea negativa.

Tabla 4. Nivel de escucha al público por parte de las organizaciones gubernamentales para publicar los anuncios de prevención de la COVID-19 según la percepción de los pobladores

\begin{tabular}{lcc}
\hline & Frecuencia & Porcentaje \\
\hline Alto & 18 & $5 \%$ \\
Regular & 35 & $9 \%$ \\
Bajo & 331 & $86 \%$ \\
\hline
\end{tabular}


José Arias Gonzáles, Tania Luz Tafur Pittman, Gloria María Delgado Suaña

Comunicación de riesgo en salud por la COVID-19 desde la percepción de los pobladores de Arequipa en 2021

De acuerdo con la Tabla 5, el 77\% de los pobladores encuestados mencionan que el nivel de planificación para publicar los anuncios de prevención de la COVID-19 es bajo. La mayoría asegura que los mensajes que le brindan no tienen objetivos claros, los cuales normalmente son confusos e incluso con información hilarante. Además, algunos anuncios contienen información que no es acorde para menores de edad o agreden algún aspecto sociodemográfico de los pobladores.

Tabla 5. Nivel de planificación de las organizaciones gubernamentales para publicar los anuncios de prevención de la COVID-19 según la percepción de los pobladores

\begin{tabular}{lcc}
\hline & Frecuencia & Porcentaje \\
\hline Alto & 21 & $5 \%$ \\
Regular & 67 & $18 \%$ \\
Bajo & 296 & $77 \%$ \\
\hline
\end{tabular}

\section{Discusión}

Como mencionan Rodríguez et al. (2018),

la labor de prevención en el nivel primario de atención en los términos de la salud, solo es posible en la comunidad si se gestan acciones efectivas de intercambio comunicativo permanente entre los especialistas y los habitantes de cada territorio. (p. 401)

Como se ha podido observar en el estudio, las organizaciones no han tenido comunicación con los pobladores a fin de crear estrategias de información para evitar el contagio, lo que ha generado una falta de credibilidad en de la población. La confianza por parte de los pobladores se ha perdido. Si bien el problema de la comunicación no es estrictamente a cargo de las organizaciones, es necesario que los pobladores también sepan informarse y busquen información veraz que pueda generarles calma y seguridad, como se evidenció en el estudio de Orozco (2020): al inicio de la pandemia, la gente solía informarse por medio de los mensajes dados por el Gobierno peruano, sin embargo, luego empezó a salir otro tipo de información por casi todos los medios de comunicación, lo que logró desorientar a las personas causando rechazo a los mensajes que vinieran de una fuente confiable o no.

Justamente por el exceso de información, mensajes con doble sentido y anuncios hilarantes han generado que las personas desestimen y desconfíen de cualquier comunicado. Al respecto, García (2017) evidencia en su estudio que a pesar de que la información llegó de forma rápida, al haber tanto contenido, la calidad de esta y el interés de la población disminuyó. Estas campañas realizadas para informar a los pobladores han recurrido a métodos de persuasión basados en provocar temor a las personas; la amenaza colectiva por el contagio ha sido una estrategia retórica que genera negación en la comunicación de riesgo en salud y una crisis para la sociedad (Prior, 2020). 


\section{Austral Omunicación \\ Volumen 10, número 2 (Diciembre de 2021): 427-440 ISSN (I) 2313-9129. ISSN (E) 2313-9137}

La respuesta de las organizaciones de la salud pública ante la pandemia por la COVID-19 ha involucrado una infodemia, ya que, en un inicio, se obligó a las personas - con el afán de cuidar su salud- a que se confinen en sus hogares y, en consecuencia, que las redes sociales se conviertan en su principal fuente de información. Esto ha generado a largo plazo lo que se muestra en este estudio y en la investigación hecha por Gonzáles et al. (2021) sobre la percepción de riesgo por la COVID-19 en pobladores de Manzanilllo. Este autor concluyó que se registra una inadecuada percepción de riesgo, que se debió a los bajos niveles de efectividad para llevar a cabo una comunicación integrada a la población y a las organizaciones a fin de evitar la enfermedad COVID-19.

\section{Conclusiones}

La comunicación de riesgo en salud por la COVID-19 se ha emitido de forma inadecuada a la población estudiada. La mayoría de los anuncios emitidos por las organizaciones responsables han generado niveles bajos de confianza en las personas, percibiendo una falta de credibilidad, actualidad, información oportuna y transparencia, además de mensajes con doble sentido que no ayudan a evitar el contagio. La importancia de escuchar al público y de planificar las acciones no han sido tomados en cuenta por parte de los medios de comunicación y esto ha repercutido en la desatención de los pobladores, provocando una negativa comunicación de riesgo en salud por la COVID-19.

Contar con estrategias de comunicación de riesgo en salud basadas en la confianza entre expertos y pobladores permitirá que haya transparencia y objetivos claros en los mensajes emitidos por los medios de comunicación con el fin de salvar vidas. Estableciendo controles de calidad en la información e indicadores de seguimiento sobre la percepción de la comunicación de riesgo se mantendrá a la población más calmada, segura y prestando atención a la orientación para evitar enfermedades o amenazas a su salud.

Debe haber un diálogo continuo entre los responsables de la salud, los medios de comunicación, las organizaciones gubernamentales y la población, no solo en tiempos de la COVID-19, sino en cualquier situación que ponga en riesgo la salud de los pobladores; este diálogo debe llevar mensajes empáticos y sinceros entendiendo el contexto en el que las personas viven. Resolver las dudas y preocupaciones de la población hace posible la planificación de procesos en la comunicación de riesgo y facilita las acciones preventivas de posibles pandemias en un futuro.

\section{Referencias}

Arias, J. L. (2020a). Proyecto de tesis, guía para la elaboración ( $1^{a}$ ed.). Enfoques Consulting. 
José Arias Gonzáles, Tania Luz Tafur Pittman, Gloria María Delgado Suaña

Comunicación de riesgo en salud por la COVID-19 desde la percepción de los pobladores de Arequipa en 2021

Arias, J. L. (2020b). Técnicas e instrumentos de investigación científica ( $1^{\mathrm{a}} \mathrm{ed}$.). Enfoques Consulting.

Brajkovic, I., Gómez-Pérez, R., Contreras, M. y Marante, D. (2020). El Coronavirus SARS-COV-2 enfermedad por Covid-19 y diabetes mellitus. Revista Venezolana de Endocrinología y Metabolismo, 18(1), 3-16. https://www.redalyc.org/ jatsRepo/3755/375564145002/html/index.html.

Feenstra, R.y Pallarés-Domínguez,D. (2017).Debates éticos en torno al neuromarketing político: el avance tecnológico y su potencial incidencia en la formación de la opinión pública. VERITAS, (35), 9-28. https://scielo.conicyt.cl/pdf/veritas/n36/ art01.pdf.

García, E. (2017). Comunicación de crisis en los gabinetes de prensa sanitarios. Análisis de la producción periodística y de la información publicada sobre la gripe A (Tesis de doctorado). Universidad Universidad Complutense de Madrid, Madrid. https://eprints.ucm.es/id/eprint/41935/1/T38575.pdf.

García-Áviles, J., Navarri-Maillo, F. y Arias-Robles, F. (2014). La credibilidad de los contenidos informativos en el Internet para los nativos digitales: caso de estudio. Palabra Clave, 17(3), 875-894. http://dx.doi.org/10.5294/pacla.2014.17.3.13.

Gobierno Regional de Arequipa. (2021). Reporte de casos confirmados de Covid19en la región de Arequipa. https://www.saludarequipa.gob.pe/epidemiologia/ Covid19/COVID19.pdf.

Gómez, L. (2017). La comunicación de riesgo en salud: aspectos teóricos y metodológicos para el control de emergencias públicas sanitaria. MEDISAN, 21(12), 7017-7030. https://www.redalyc.org/pdf/3684/368454498013.pdf.

Gonzáles, J., Soler, Y., Pérez, E., Gonzáles, R. y Pons, S. (2021). Percepción de riesgo ante la COVID-19 en pobladores del municipio Manzanillo. Multimed. Revista Médica, 25(1),

Hernández, J., Garbey, E. y Enríquez, I. (2021). Importancia de la perspectiva afectiva e intercultural en la comunicación profesional en la comunicación profesional. Revista Habanera de Ciencias Médicas, 20(1).

Hernández-Sampieri, R.(2019). Metodología de la investigación(7 ed.). McGraw-Hill. Lázaro-Rodríguez, P. y Herrera-Viedma, E. (2020). Noticias sobre Covid-19 y 2019$\mathrm{nCoV}$ en medios de comunicación de España: el papel de los medios digitales en tiempos de confinamiento. El profesional de la información , 29(3), 1-11. http:// profesionaldelainformacion.com/contenidos/2020/may/lazaro-herrera.pdf.

Martínez, S. (2017). Uso y abuso del término percepción de riesgo. Revista Cubana de Salud Pública, 43(3), 412-419. https://www.scielosp.org/pdf/rcsp/2017. v43n3/412-418/es.

Masip, P.,Aran-Ramspott, S., Ruiz-Caballero, C., Suau, J., Almenar, E.y Puertas-Graell, 


\section{Austral@municación \\ Volumen 10, número 2 (Diciembre de 2021): 427-440 ISSN (I) 2313-9129. ISSN (E) 2313-9137}

D. (2020). Consumo informativo y cobertura mediática durante el confinamiento por el Covid-19: sobreinformación, sesgo ideólogico y sensacionalismo. El profesionalismo de la información , 29(3), 1-12. https://doi.org/10.3145/epi.2020. may.12.

Mejia, C., Rodríguez-Alarcon, J., Enríquez, M., Moreno, A., Huaytán-Rojas, K., Huancahuari-Ñeñacc, N., Julca-Gonzales, A., Álvarez, C., Choque-Vargas, J. y Curioso, W. (2020). Percepción de miedo o exageración que transmiten los medios de comunicación en la población peruana durante la pandemia de la COVID-19. Revista Cubana de Investigaciones Biomédicas, 39(2), e698. http://scielo.sld.cu/ pdf/ibi/v39n2/1561-3011-ibi-39-02-e698.pdf.

Ministerio de Salud de Chile. (2020). Manual de comunicación de riesgo en emergencias $y$ desastres naturales. https://degreyd.minsal.cl/wp-content/uploads/2018/04/ MANUAL-COMUNICACION-DE-RIESGO-MINSAL-Versi\%C3\%B3n-1.0-18diciembre-2020.pdf.

Organización Mundial de la Salud. (2017). Comunicating for health. https://www.who. int/about/communications.

Organización Panamericana de la Salud. (2011). Guía para la elaboración de estrategias de comuniación de riesgo. https://www.paho.org/es/documentos/ guia-para-elaboracion-estrategia-comunicacion-riesgo-teoria-accion-2011.

Orozco, C. (2020). Estrategias de comunicación del gobierno del Perú durante el Estado de Emergencia por la Covid-19. Estudio de Caso: Mensaje a la Nación del presidente Martín Vizacarra emitido por el medio estatal TV Perú en el periodo Marzo-Abril de 2020. (Tesis de bachiller). Universidad San Ignacio de Loyola, Lima. http://repositorio.usil.edu.pe/bitstream/USIL/11146/1/2020_Orozco\%20 Ayala.pdf.

Peña-López, B. y Rincón-Orozco, B. (2020). Generalidades de la Pandemia por COVID-19 y su asociación genética con el virus del SARS. Salud UIS, 52(2), 83-87. https://revistas.uis.edu.co/index.php/revistasaluduis/article/view/10639/10446.

Prior, H. (2020). Comunicación pública de riesgo en tiempo de pandemia: las respuesta de Portugar a la Covid-19. Más Poder Local, (41), 6-11. https://dialnet.unirioja.es/ servlet/articulo?codigo $=7407213$.

Rodríguez, C. (2019). No diga fake news, di desinformación: una revisión sobre el fenómeno de las noticias falsas y sus implicaciones. Comunicación, (40), 65-74. https://dialnet.unirioja.es/servlet/articulo?codigo $=7028909$.

Rodríguez, S., Cabrera, L. y Calero, E. (2018). La comunicación social en salud para la prevención de enfermedades en la comunidad. Revista Humnanidades Médicas, 18(2), 384-404. http://scielo.sld.cu/scielo. php?script=sci_abstract\&pid=S1727-81202018000200384. 
José Arias Gonzáles, Tania Luz Tafur Pittman, Gloria María Delgado Suaña

Comunicación de riesgo en salud por la COVID-19 desde la percepción de los pobladores de Arequipa en 2021

Rosas, M. y Barrios, A. (2017). Comunicación de riesgo, cambio climático y crisis ambientales. Revista Latinoamericana de Comunicación, (136), 179-194. https:// dialnet.unirioja.es/servlet/articulo? codigo $=6242352$. 